\title{
La influencia del tipo de tarea en la fluidez $y$ la exactitud léxica de la producción escrita de aprendientes griegos de español como lengua extranjera
}

\author{
Kiriakí Palapanidi e Irini Mavrou \\ Universidad Antonio de Nebrija
}

Recibido: 21 de noviembre de 2012 / Aceptado: 25 de enero de 2013

ISSN: $1697-7467$

\begin{abstract}
RESUMEN: El objetivo del presente estudio es examinar la influencia del tipo de tarea (narración - argumentación) en la fluidez y la exactitud léxica de la producción escrita de aprendientes griegos de español como lengua extranjera así como la posible interacción entre ellas dentro del mismo tipo de tarea. Los resultados han mostrado que el tipo de tarea influye tanto en la fluidez como en la exactitud léxica. En concreto, se ha observado mayor fluidez pero menor exactitud léxica en la tarea narrativa. Adicionalmente, se han observado correlaciones significativas entre la fluidez y la exactitud léxica solo en la tarea narrativa. Los resultados se explican basándose en el Modelo de la Capacidad Limitada de Atención de Skehan y la Hipótesis de Cognición de Robinson.

Palabras clave: narración escrita, argumentación escrita, fluidez de expresión escrita, exactitud léxica de expresión escrita
\end{abstract}

The Effect of Task Type on Fluency and Lexical Accuracy of Greek SFL (Spanish Foreign Languge) Learners' Written Performance

\begin{abstract}
This research examines the effect of task type (narration - argumentation) on fluency and lexical accuracy in written production of Greek students of Spanish as a foreign language and the possible interaction between them in the same task type. The results have shown that task type has an impact on fluency and lexical accuracy. More precisely, greater fluency but less lexical accuracy in the narrative task has been observed. Additionally, the results have shown significant correlations between the fluency and the lexical accuracy only in the narrative task. The results are discussed in the light of Skehan's Limited Attentional Capacity Model and Robinson's Cognition Hypothesis.

Keywords: written narration, written argumentation, fluency in written composition, lexical accuracy in written composition
\end{abstract}

\section{INTRODUCCIÓN}

La fluidez y la exactitud (o corrección) son dos conceptos fundamentales en la Didáctica y la Adquisición de Segundas Lenguas (ASL). El Marco Común Europeo de Referencia para las Lenguas (MCER) señala que se trata de dos componentes de la competencia comunicativa en lengua extranjera (LE). Según el MCER, la fluidez es uno de los componentes de la 
competencia funcional que, junto con la competencia discursiva y organizativa, forma parte de la competencia pragmática. El MCER la define como "la capacidad de articular, de seguir adelante y de desenvolverse bien cuando se llega a un callejón sin salida" (p.125), mientras que en el Diccionario de términos clave de español como lengua extranjera del Centro Virtual Cervantes la fluidez se define como "la habilidad de procesar (tanto en su recepción como en su producción) la lengua -aspectos semánticos, morfosintácticos, socioculturales, etc.- con soltura y coherencia, sin excesivas pausas o titubeos y a una velocidad equiparable o próxima a la de los hablantes nativos". Desde este punto de vista, el término de fluidez se refiere tanto a la fluidez verbal (oral y escrita) como a la fluidez lectora.

Por otro lado, el concepto de exactitud, como señalan Housen y Kuiken (2009), se considera el más transparente y el más antiguo. Según el Diccionario de términos clave de español como lengua extranjera del Centro Virtual Cervantes, la exactitud lingüística concierne a "la ausencia de errores en el uso de cualquiera de las destrezas lingüísticas", y atañe tanto a la gramática como a otros componentes del sistema lingüístico (el léxico, la ortografía, etc.).

Los dos conceptos mencionados anteriormente se han tratado en la investigación de la ASL desde diferentes perspectivas. Según Simensen (2010), se han utilizado como objetivos de la enseñanza de LE y como criterios de evaluación de la producción lingüística en LE. Housen y Kuiken (2009), a su vez, añaden que tanto la precisión como la fluidez han sido empleadas como medidas del desarrollo lingüístico en LE, como descriptores de la actuación en la producción escrita y oral y como indicadores del nivel lingüístico en LE.

El presente estudio se centra en la fluidez y la exactitud de la producción escrita de aprendientes griegos de español como lengua extranjera (ELE). Considera estos conceptos componentes de la competencia comunicativa y los utiliza como descriptores de la expresión escrita con el fin de observar la influencia que puede ejercer el tipo de tarea escrita en dichos descriptores así como su posible interacción dentro del mismo tipo de tarea escrita.

\section{LA TAREA COMO FACTOR CONDICIONANTE DE LA PRODUCCIÓN LINGÜÍSTICA (ORAL Y ESCRITA) EN LENGUA EXTRANJERA}

Según Agustín Llach, Espinosa y Fontecha (2005), el que la producción lingüística de los estudiantes de LE varíe de acuerdo a la tarea que tienen que realizar, es un tema bien conocido en el campo de la ASL. Varios son los estudiosos que afirman este hecho (Tarone, 1983; Carrell y Connor, 1991; Larsen-Freeman y Long, 1991). Los dos modelos que han tratado de describir y explicar la relación entre la producción lingüística en LE y las características de la tarea son el Modelo de la Capacidad Limitada de Atención, que se describe en los trabajos de Skehan (1998) y de Skehan y Foster $(1999,2001)$ y la Hipótesis de Cognición, explicada en los trabajos de Robinson $(2001,2007)$.

\subsection{El Modelo de la Capacidad Limitada de Atención}

La investigación de Skehan (1998) y Skehan y Foster $(1999,2001)$ se centra en la tarea desde una perspectiva influenciada por las teorías del procesamiento de la información en el aprendizaje de LE. En concreto, Skehan y Foster $(1999,2001)$ se basan en los trabajos 
de VanPatten $(1985,1989,1990)$ para proponer un modelo propio que persigue describir la relación entre las demandas de atención de las tareas y la actuación lingüística de los aprendices de LE.

VanPatten (1989) sostiene que la capacidad de procesar la información es limitada y que el procesamiento consciente de ésta se realiza de manera sucesiva. Por este motivo, los aprendientes de LE de los niveles iniciales e intermedios no pueden centrarse conscientemente en la forma del input y tampoco pueden procesar simultáneamente el significado y la forma. Esto puede ocurrir sólo cuando se automatiza la habilidad de comprensión. Sin embargo, señala que los elementos lingüísticos dotados de información significativa, como los elementos léxicos, se pueden procesar en todos los niveles.

Bajo la influencia de esta teoría, Skehan y Foster $(1999,2001)$ proponen el Modelo de la Capacidad Limitada de Atención. Según este modelo, los aprendientes de LE no pueden atender de igual manera a todas las dimensiones de la lengua debido justamente a su capacidad limitada de atención. Por tanto, la selección del aspecto al que decidan dar prioridad determinará su actuación.

Skehan (1996) afirma que las tareas son actividades en las que se da prioridad al significado. Durante la actuación, el énfasis se pone en la importancia de cumplir el objetivo comunicativo, relegándose a un segundo plano aspectos de la lengua como, por ejemplo, la corrección gramatical. El foco en el significado al llevar a cabo tareas de aprendizaje y la Capacidad Limitada de Atención de los aprendientes de LE hacen que éstos presten más atención al contenido de la tarea, de modo que otros aspectos lingüísticos de su output se ven afectados negativamente.

En resumen, la premisa básica que subyace al Modelo de la Capacidad Limitada de Atención es que a medida que aumenta la complejidad cognitiva de la tarea, la atención se dirige hacia el significado de la producción lingüística. Teniendo en cuenta la distinción de VanPatten $(1985,1989,1990)$ entre el foco en el significado y el foco en la forma, la fluidez se relaciona con el foco en el significado, mientras que la exactitud lingüística se vincula con el foco en la forma.

\subsection{La Hipótesis de Cognición}

Robinson $(2001,2007)$ propone un modelo diferente para explicar la relación entre las características de la tarea y la producción lingüística en LE: el Marco Triádico Componencial. Mediante dicho modelo el autor pretende formular una base teórica para la secuenciación de las tareas de aprendizaje y para el estudio de la influencia de sus características en la producción lingüística en LE.

Robinson (2001) establece algunos criterios que permiten secuenciar las tareas según sus requerimientos cognitivos. Dichos criterios se dividen en tres grupos: los que se relacionan con la complejidad de la tarea, donde se incluyen los factores cognitivos; los que influyen en la percepción de la dificultad de la tarea, donde se incluyen los factores del aprendiente, sean éstos cognitivos o afectivos, y, por último, los relacionados con las condiciones de la tarea, en los que están incorporados los factores interaccionales.

En cuanto a los factores cognitivos, que se estudian en el presente trabajo, Robinson (2007) los agrupa en dos dimensiones. La dimensión resource-directing, que se refiere a aquellas variables (aquí y ahora versus allí y entonces, cantidad de elementos, razonamiento 
espacial, causal e intencional y toma de perspectiva) que dirigen los recursos atencionales hacia los aspectos lingüísticos y la dimensión resource-dispersing que comprende aquellos factores (tiempo de planificación, conocimiento previo, tarea simple versus tarea dual, estructura de la tarea, número de pasos e independencia de pasos) cuya manipulación resulta en una dispersión de los recursos atencionales y de memoria.

Robinson (2007) sostiene que en las tareas que suponen una mayor carga cognitiva se emplean más recursos cognitivos, hecho que influye tanto en la producción lingüística, en el output de los aprendices de LE, como también en la manera en que éstos utilizan el input. Cuando una tarea implica altos requerimientos cognitivos, el aprendiente se ve obligado a modificar su output con el objetivo de cumplir estas demandas, y trata de basarse en los conocimientos de los que dispone para superar las dificultades que se le plantean, tendiendo así a incorporar más formas del input.

En base a lo anterior, Robinson $(2001,2007)$ formula la Hipótesis de Cognición. Según esta hipótesis, a medida que aumentan los requerimientos cognitivos de una tarea manipulando las variables de la dimensión resource-directing, el aprendiente de LE -a fin de superar las mayores demandas funcionales y comunicativas- produce un discurso más exacto y complejo, aunque en detrimento de la fluidez. Por otro lado, el aumento de la complejidad en la dimensión resource-dispersing lleva a una dispersión de los recursos atencionales dificultando el acceso eficaz al sistema de interlengua, lo cual influye negativamente en todas las dimensiones de la lengua.

\subsection{La investigación de la influencia del tipo de tarea en la fluidez y la exactitud léxica de la producción escrita en LE}

La investigación sobre la manipulación cognitiva de las tareas y su influencia en la producción lingüística en LE ha sido posible gracias a los intentos de los especialistas, anteriormente citados, de determinar las características de las tareas de modo de poder manipularlas y estudiarlas de forma empírica. Sus planteamientos constituyeron un punto de partida de numerosos estudios empíricos en el campo de la ASL, en los que se examina cómo la manipulación de ciertas características de una tarea incide en aspectos como la fluidez, la exactitud y la complejidad lingüística. Siendo los dos primeros aspectos objeto de interés del presente estudio, a continuación, se presentan algunas investigaciones que enfocaron en estos en relación con el discurso escrito en LE.

Way, Joiner y Seaman (2000) examinaron la influencia del tipo de tarea en la fluidez de la producción escrita en LE de aprendientes de francés como LE. Éstos realizaron tres tareas de producción escrita (descriptiva, narrativa y expositiva) y su actuación fue evaluada en términos de calidad general, fluidez, complejidad sintáctica y exactitud. De acuerdo con los resultados arrojados por este estudio, en la tarea descriptiva se logró mejor calidad general e índices de fluidez y exactitud lingüística más altos en comparación con la tarea narrativa. Por otro lado, los índices más bajos de fluidez y exactitud léxica así como de calidad general se observaron en la tarea expositiva. En lo referente a la complejidad sintáctica, no hubo diferencias significativas entre los tres tipos de tarea.

Rezazadeh, Tavakoli y Rasekh (2011) emprendieron un estudio cuyo objetivo era investigar la manera en que varían tres aspectos de la producción escrita de aprendientes iraníes de inglés como LE (fluidez, complejidad y exactitud) a partir de dos diferentes tipos de 
tarea de producción escrita (instrucción y argumentación). Los resultados mostraron mayor fluidez y exactitud en la tarea que requería proporcionar instrucciones y mayor complejidad sintáctica en la tarea argumentativa.

Por último, Agustín Llach, Moreno Espinosa y Fernández Fontecha (2005) examinaron la variación cuantitativa y cualitativa de los errores léxicos en dos tipos de tarea escrita, una descriptiva y otra narrativa. Los resultados obtenidos mostraron que el tipo de tarea escrita tuvo un impacto en la exactitud léxica. En concreto, los informantes de su estudio cometieron más errores léxicos en la tarea narrativa que en la tarea descriptiva. Además, se observó que en los dos tipos de tarea escrita se habían cometido diferentes tipos de errores léxicos.

En este apartado se han presentado algunos estudios sobre la influencia del tipo de tarea escrita en la fluidez y la exactitud léxica. Cabe señalar, no obstante, que en el campo de la ASL la mayor parte de la bibliografía concerniente a la influencia del tipo de tarea en la producción lingüística está centrada en la producción oral. Por lo tanto, la influencia del tipo de tarea escrita en la actuación, objeto de interés del presente estudio, constituye un área científica abierta para mayor investigación.

\section{Metodología}

Teniendo como base las teorías expuestas, a continuación se concretan los objetivos del presente estudio y se formulan las hipótesis de trabajo. Asimismo, se describe la metodología empleada para la realización del mismo exponiendo los procedimientos que se han seguido para la recogida y el consiguiente procesamiento de los datos.

\subsection{Objetivos del estudio, preguntas e hipótesis de investigación}

El objetivo del presente estudio consiste en examinar la influencia del tipo de tarea (narración - argumentación) en la fluidez y la exactitud léxica de la producción escrita de aprendientes griegos de ELE. Adicionalmente, se persigue examinar la interacción entre la fluidez y la exactitud léxica en el mismo tipo de tarea escrita.

Para ello se han formulado las siguientes preguntas e hipótesis de investigación.

1. ¿Influye el tipo de tarea en la fluidez de la producción escrita?

2. ¿Influye el tipo de tarea en la exactitud léxica de la producción escrita?

3. ¿Existe alguna relación entre la fluidez y la exactitud léxica dentro del mismo tipo de tarea de producción escrita?

Las teorías citadas permiten la formulación de las siguientes hipótesis de trabajo.

1. El tipo de tarea escrita influye en la fluidez de la producción escrita.

2. El tipo de tarea escrita influye en la exactitud léxica de la producción escrita.

3. Hay interacción entre la fluidez y la exactitud léxica dentro del mismo tipo de tarea de producción escrita. 


\subsection{Participantes}

La muestra, compuesta de 47 aprendientes griegos de ELE con un nivel de dominio lingüístico B2 según el MCER, incluía a alumnos adultos del Centro de Idiomas Extranjeros de la Universidad Kapodistríaca de Atenas, cuya lengua materna era el griego. Además, el hecho de ser aprendientes de ELE en un contexto institucionalizado, suponía un conocimiento explícito de la lengua y un input controlado y totalmente dependiente de lo que enseñan sus profesores en clase.

\subsection{Instrumentos de recogida de datos}

Dados los objetivos del presente trabajo, se les asignaron a los participantes dos tareas de producción escrita: una narración y una argumentación. La primera tarea consistía en la narración de la última película que habían visto, mientras que en la segunda los informantes tenían que expresar su punto de vista sobre la vida en las pequeñas ciudades y en las grandes capitales razonando su opinión (Figura 1).

1) Probablemente eres aficionado al cine. ¿Recuerdas la última película que has visto? Cuéntala y habla de: dónde, cuándo y cómo se inicia la historia; qué sucede posteriormente; cuál es el momento más importante; cómo termina.

2) En la actualidad vivir en pequeñas ciudades, cerca de la naturaleza, ofrece más ventajas que la vida en las grandes capitales. Elabora un escrito en el que deberás: exponer tu opinión a favor o en contra; dar ejemplos que justifiquen tu opinión; hablar de tu experiencia personal; elaborar una breve conclusión.

Figura 1. Tareas de producción escrita

Los dos tipos de tarea mencionados activan diferentes procesos cognitivos y, por ello, imponen diferente carga cognitiva. La tarea narrativa se localiza temporalmente en el pasado y requiere la operación de la memoria -ya que los participantes carecen de apoyo contextual-, por tanto, se puede considerar de alta complejidad según el modelo de Robinson (2007). En cuanto a la tarea argumentativa, pese a la necesidad de proporcionar argumentos y razonar -lo cual seguramente la convierte en una tarea compleja-, es importante señalar que la información que tenían que procesar los informantes del presente estudio se localiza en el aqui y ahora. Ello significa que los aprendices debían emplear un tiempo verbal ampliamente practicado durante sus años de estudio del español y en gran medida automatizado, es decir, el presente de indicativo. Cabe agregar que el tema de la tarea argumentativa utilizada se relaciona con la vida cotidiana de los aprendices, pudiéndose asumir, entonces, que dicha tarea es de menor complejidad ya que los participantes pueden expresarse más libremente, utilizando un vocabulario más familiar, perteneciente al campo semántico de sus intereses personales.

\subsection{Procedimiento de recogida de datos}

La recogida de datos se realizó en el Centro de Idiomas Extranjeros de la Universidad de Atenas durante la hora de clase y en presencia del profesor. Los participantes dispusieron 
de 50 minutos para elaborar las redacciones sin que se les hubiera impuesto un límite mínimo ni máximo de palabras. Tampoco podían recurrir a material de apoyo, como gramáticas o diccionarios.

\subsection{Procesamiento de los textos escritos}

Teniendo en cuenta los objetivos del presente estudio, los textos se procesaron en términos de fluidez y exactitud léxica. Para ello se emplearon diferentes medidas generales utilizadas en estudios afines. Concretamente, para medir la fluidez de la producción escrita se optó por tres índices: a) el número de palabras, b) el número de cláusulas y c) el número de unidades T (Wigglesworth y Storch, 2009; Rezazadeh, Tavakoli y Rasekh, 2011).

En lo referente a la exactitud, es importante señalar que en estudios similares (Zhang, 1987; Rezazadeh, Tavakoli y Rasekh, 2011; Serrano, 2011) su medición se hizo en función de diferentes tipos de errores lingüísticos (sintácticos, morfológicos, ortográficos o errores de puntuación). Dado que el presente estudio enfoca solo en la exactitud léxica, se tuvieron en cuenta únicamente los errores léxicos. El método de investigación que se utilizó para identificar dichos errores fue el Análisis de Errores.

Se calculó el número de errores léxicos, formales y semánticos. Entre los errores formales se han distinguido los siguientes tipos: selección errónea del género, selección errónea del número, extranjerismo, cambio de código, uso de un significante español próximo, creación de palabras inexistentes en LE. En cuanto a los errores semánticos, se han incluido los siguientes casos: lexemas con semas comunes pero no intercambiables en el contexto, traducción literal, falsos amigos, registro no apropiado a la situación, pleonasmo, cambios entre derivados de la misma raíz, perífrasis, errores en el uso de ser-estar, colocaciones. Además, se han calculado tanto los errores léxicos debidos a la interferencia de la lengua materna u otra L3 como los debidos a las idiosincrasias del sistema lingüístico español.

\section{Resultados}

En este apartado se presentan los datos descriptivos y los procedimientos estadísticos llevados a cabo a fin de alcanzar los objetivos planteados; esto es, examinar la potencial influencia de los dos tipos de tarea de escritura en la cantidad de discurso producido y en el grado de precisión léxica logrado, así como la posible interacción entre la fluidez y la exactitud léxica de la producción escrita dentro del mismo tipo de tarea.

\subsection{Estadística descriptiva}

Las Tablas 1 y 2 recogen los datos descriptivos respecto a las variables seleccionadas. Los resultados revelan que las medias de las tres medidas de fluidez son mayores en las composiciones de tipo narrativo que en las de tipo argumentativo. En cuanto a la exactitud léxica, la presencia de errores léxicos parece ser más fuerte en las redacciones de tipo narrativo. 
Tabla 1. Medidas de fluidez por tipo de tarea

\begin{tabular}{|l|c|c|c|c|c|c|c|c|c|c|c|c|}
\hline \multirow{3}{*}{$\begin{array}{c}\text { Tipo de tarea } \\
\text { escrita }\end{array}$} & \multicolumn{10}{|c|}{ Medidas de fluidez } \\
\cline { 2 - 15 } & \multicolumn{4}{|c|}{ Número de palabras } & \multicolumn{4}{c|}{ Número de cláusulas } & \multicolumn{3}{c|}{ Número de unidades T } \\
\cline { 2 - 13 } & Med. & D.T. & Mín. & Máx. & Med. & D.T. & Mín. & Máx. & Med. & D.T. & Mín. & Máx. \\
\hline Narración & 163,23 & 56,99 & 63 & 293 & 20,87 & 9,08 & 8 & 52 & 9,98 & 4,94 & 3 & 32 \\
\hline Argumentación & 142,79 & 40,06 & 71 & 244 & 16,6 & 4,77 & 8 & 28 & 8,04 & 2,41 & 5 & 16 \\
\hline
\end{tabular}

Tabla 2. Medida de exactitud léxica por tipo de tarea

\begin{tabular}{|l|c|c|c|c|}
\hline \multirow{2}{*}{$\begin{array}{l}\text { Tipo de tarea } \\
\text { escrita }\end{array}$} & \multicolumn{4}{|c|}{ Medida de exactitud léxica } \\
\cline { 2 - 5 } & Media & D.T. & Valores mínimos & Valores máximos \\
\cline { 2 - 5 } & 5,57 & 3,97 & 0 & 15 \\
\hline Narración & 4,4 & 2,82 & 0 & 13 \\
\hline Argumentación & & \multicolumn{4}{|c|}{} \\
\hline
\end{tabular}

\subsection{Análisis estadísticos}

Para el procesamiento estadístico de los datos, en primer lugar se aplicó la prueba de normalidad Shapiro-Wilk mediante la cual se constató que los datos obtenidos no presentan una distribución teórica normal, excepto en el caso de la cantidad de palabras, hecho que obligó al uso de análisis estadísticos tanto paramétricos como no paramétricos. De este modo, y teniendo en cuenta las dos primeras preguntas de investigación, se utilizó la prueba $\mathrm{T}$ para muestras relacionadas al analizar los datos que presentaron normalidad, y la prueba de Wilcoxon en los casos en los que no se cumplió este criterio. En cuanto a la tercera pregunta de investigación, es decir, la existencia o no de relación entre fluidez y exactitud léxica, se utilizó el coeficiente de correlación de Spearman de rangos ordenados. Para todos los análisis realizados se estableció un nivel de significancia de $\alpha=0,05$.

4.2.1. Influencia del tipo de tarea en la fluidez y la exactitud léxica de la producción escrita

En las Tablas 3 y 4 se presentan los resultados obtenidos tras realizar los análisis estadísticos correspondientes para determinar la posible influencia del tipo de tarea en la fluidez y la exactitud léxica. En cuanto al número total de palabras producido, la prueba $\mathrm{T}$ (Tabla 3) reveló diferencias estadísticamente significativas entre narración y argumentación $(\mathrm{t}(46)=3,130, \mathrm{p}<0,05)$. 
Tabla 3. Comparación del número total de palabras entre los dos tipos de tarea

\begin{tabular}{|c|c|c|c|c|c|c|c|c|c|}
\hline \multicolumn{10}{|c|}{ Prueba $\mathrm{T}$ de muestras relacionadas } \\
\hline & & \multicolumn{5}{|c|}{ Diferencias relacionadas } & \multirow[t]{3}{*}{$\mathrm{t}$} & \multirow[t]{3}{*}{$\mathrm{gl}$} & \multirow{3}{*}{$\begin{array}{c}\text { Sig. } \\
\text { (bilateral) }\end{array}$} \\
\hline & & \multirow[t]{2}{*}{ Media } & \multirow[t]{2}{*}{ D. T. } & \multirow{2}{*}{$\begin{array}{l}\text { Error } \\
\text { típ. } \\
\text { de la } \\
\text { media }\end{array}$} & \multicolumn{2}{|c|}{$\begin{array}{l}95 \% \text { Intervalo } \\
\text { de confianza para la } \\
\text { diferencia }\end{array}$} & & & \\
\hline & & & & & Inferior & Superior & & & \\
\hline Par 1 & $\begin{array}{c}\text { Palabras } \\
\text { Narración } \\
\text { - Palabras } \\
\text { Argumentación }\end{array}$ & 20,447 & 44,779 & 6,532 & 7,299 & 33,594 & 3,130 & 46 & 0,003 \\
\hline
\end{tabular}

Se obtienen resultados parecidos respecto al resto de los índices lingüísticos utilizados para medir la fluidez (Tabla 4). Según los resultados arrojados por la prueba de Wilcoxon, existen diferencias significativas tanto en el número total de cláusulas $(Z=-3,324, p<0,05)$ como en el número de unidades $\mathrm{T}(\mathrm{Z}=-3,201, \mathrm{p}<0,05)$ entre las dos tareas de producción escrita empleadas en el presente estudio. Por lo tanto, se puede asumir que el tipo de tarea influyó en la fluidez, siendo la tarea narrativa la que elicitó mayor fluidez.

En lo referente a la otra variable examinada, los resultados de la prueba de Wilcoxon (Tabla 4) muestran que la exactitud léxica alcanzada por los informantes se diferencia de manera significativa entre las dos tareas $(Z=-2,198, p<0,05)$. Dicho de otro modo, en la tarea argumentativa se dio mayor exactitud léxica en comparación con la narración.

Tabla 4. Comparación de cláusulas, unidades $T$ y errores léxicos entre los dos tipos de tarea

Estadísticos de contraste ${ }^{\mathrm{a}}$

\begin{tabular}{|l|c|c|c|}
\hline & $\begin{array}{c}\text { Cláusulas Argumentación } \\
- \text { Cláusulas Narración }\end{array}$ & $\begin{array}{c}\text { Unidades T Argumentación } \\
- \text { Unidades T Narración }\end{array}$ & $\begin{array}{c}\text { Errores léxicos Argumentación } \\
- \text { Errores léxicos Narración }\end{array}$ \\
\hline $\mathrm{Z}$ & $-3,324^{\mathrm{b}}$ & $-3,201^{\mathrm{b}}$ & $-2,198^{\mathrm{b}}$ \\
\hline Sig. asintót. (bilateral) & 0,001 & 0,001 & 0,028 \\
\hline
\end{tabular}

a. Prueba de los rangos con signo de Wilcoxon

b. Basado en los rangos positivos

\subsubsection{Relación entre fluidez y exactitud léxica de la producción escrita dentro del mismo tipo de tarea}

Con la intención de examinar posibles asociaciones entre la fluidez y la exactitud léxica dentro del mismo tipo de tarea se procedió a un análisis de correlación de Spearman. Los resultados obtenidos para la narración se presentan en la Tabla 5, mientras que los referidos a la argumentación en la Tabla 6. 
De dicho análisis se infiere que todas las medidas de fluidez utilizadas correlacionan entre sí de modo significativo $(\mathrm{p}<0,01)$ y para ambos tipos de tarea, por lo tanto, se puede decir que dichas medidas reflejan el mismo constructo. En cuanto a la tarea narrativa (Tabla 5 ), los resultados muestran asociaciones positivas moderadas entre todas las medidas de fluidez y el número total de errores léxicos que cometieron los informantes, lo cual parece indicar que a medida que aumenta la fluidez la exactitud léxica tiende a disminuir.

Tabla 5. Correlación de Spearman entre las variables examinadas en la tarea narrativa

\begin{tabular}{ccccc}
\hline Variables & 1 & 2 & 3 & 4 \\
\hline $\begin{array}{l}\text { 1. Palabras } \\
\text { Narración }\end{array}$ & - & & & \\
$\begin{array}{l}\text { 2. Cláusulas } \\
\text { Narración }\end{array}$ & $0,903^{* *}$ & - & & \\
3.Unidades T & $0,765^{* *}$ & $0,831^{* *}$ & - & - \\
$\quad$ Narración & & & & \\
4. Errores léxicos & $0,382^{* *}$ & $0,404^{* *}$ & $0,432^{* *}$ & \\
$\quad$ Narración & & & &
\end{tabular}

** La correlación es significativa al nivel $\mathrm{p}<0,01$ (bilateral).

Una imagen diferente se obtiene en relación con la argumentación. En la Tabla 6 se observa una falta de asociación entre dos de los índices de fluidez examinados -número de palabras y de unidades T-, y la exactitud léxica. La única correlación estadísticamente significativa concierne al número de cláusulas con el número de errores léxicos que hicieron

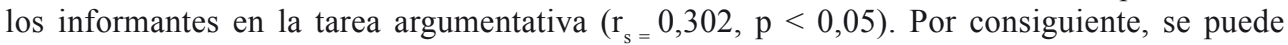
afirmar que producir un discurso más fluido no conlleva necesariamente la producción de un texto más o menos correcto en términos de exactitud léxica.

Tabla 6. Correlación de Spearman entre las variables examinadas en la tarea argumentativa

\begin{tabular}{|c|c|c|c|c|}
\hline Variables & 1 & 2 & 3 & 4 \\
\hline $\begin{array}{l}\text { 1. Palabras } \\
\text { Argumentación }\end{array}$ & - & & & \\
\hline $\begin{array}{l}\text { 2. Cláusulas } \\
\text { Argumentación }\end{array}$ & $0,748 * *$ & - & & \\
\hline $\begin{array}{l}\text { 3.Unidades T } \\
\text { Argumentación }\end{array}$ & $0,551 * *$ & $0,616 * *$ & - & \\
\hline $\begin{array}{l}\text { 4. Errores léxicos } \\
\text { Argumentación }\end{array}$ & 0,195 & $0,302^{*}$ & 0,137 & - \\
\hline
\end{tabular}




\section{Discusión}

El principal objetivo del presente estudio fue examinar la influencia del tipo de tarea en la fluidez y la exactitud léxica de la producción escrita de estudiantes griegos de ELE. Además, se persiguió examinar la posible interacción entre fluidez y exactitud léxica dentro del mismo tipo de tarea. Para cumplir estos objetivos, se emplearon dos tipos de tarea de producción escrita de diferentes grados de complejidad cognitiva (narración y argumentación), y los textos se procesaron en términos de fluidez y exactitud léxica.

La discusión de los hallazgos obtenidos respeta el orden de formulación de las preguntas de partida. De este modo, en cuanto a las dos primeras preguntas de investigación, se observó una diferencia estadísticamente significativa en las medidas empleadas tanto de fluidez como de exactitud léxica entre los dos tipos de tareas de escritura, lo cual indica que el tipo de tarea tuvo efectivamente un impacto en la actuación de los participantes.

Los hallazgos encontrados coinciden con estudios similares que han mostrado que el tipo de tarea escrita influye en estos dos aspectos de la actuación. Way, Joiner y Seaman (2000), que analizaron la fluidez de tres tipos diferentes de tarea escrita (descripción, narración, exposición), descubrieron que la cantidad de discurso producido en cada tipo de tarea escrita variaba. Asimismo, los resultados arrojados por Rezazadeh, Tavakoli y Rasekh (2011) indicaron diferencias significativas en la fluidez de los dos tipos de tarea escrita examinados (instrucción, argumentación). En cuanto a la exactitud léxica, en el trabajo de Agustín Llach, Espinosa y Fontecha (2005) se observó diferencia en la producción cuantitativa de errores léxicos en los dos tipos de tarea escrita analizados (descripción, narración).

Más en concreto, los resultados arrojados por el presente estudio mostraron que la tarea que supuso un mayor grado de complejidad cognitiva, es decir, la narrativa elicitó mayor fluidez que la argumentación aunque no ocurrió lo mismo respecto al número de errores léxicos cometidos. En este caso fue en la tarea argumentativa donde los informantes consiguieron un grado más elevado de exactitud léxica. Estos resultados parecen contradecir los postulados de la Hipótesis de Cognición, según la cual el aumento de la complejidad cognitiva en la dimensión resource-directing a la que pertenece la variable aqui y ahora, si bien favorece la exactitud y la complejidad lingüística, influye negativamente en la fluidez.

Una posible explicación del resultado del presente estudio probablemente reside en una de las características del género argumentativo. Toda argumentación precisa cierto grado de razonamiento, otra de las variables que Robinson (2007) clasifica en la dimensión resourcedirecting. Teniendo esto en cuenta, es posible pensar que el grado de razonamiento exigido por la tarea argumentativa seguramente tiene un mayor impacto en la actuación que la manipulación de la variable aquí y ahora en sí. En otras palabras, puede que la tarea argumentativa utilizada en el presente estudio impusiera mayor carga cognitiva que la narración $\mathrm{y}$, por tanto, que en esta tarea prevaleciera la canalización de los recursos cognitivos de los participantes más hacia los aspectos lingüísticos. Desde este punto de vista, los resultados apoyarían la Hipótesis de Cognición.

La mayor fluidez en la tarea narrativa en comparación con la tarea argumentativa fue expuesta, también, por Rashid y Rafik-Galea (2007). Los investigadores analizaron la calidad general y la fluidez de dos tipos de tarea escrita (narración, argumentación) de aprendientes malasios de inglés como LE. Sus resultados mostraron que los participantes tendieron a producir textos más largos en las redacciones de tipo narrativo que en las de tipo argumentativo, lo cual vendría a confirmar el resultado de la presente investigación. 
Por lo que respecta a la exactitud léxica, la fuerte presencia de errores léxicos en la tarea narrativa se puede interpretar en base a la Hipótesis de Cognición, y, más en concreto, atendiendo a una de las variables que recaen en la dimensión resource-dispersing, esto es, el grado de familiaridad de la información procesada o el conocimiento previo, como lo denomina Robinson. Como afirma el autor, la familiaridad con el tema puede facilitar la actuación de los aprendientes de LE (Robinson, 2007). De hecho, se observa que en la narración, donde se requería transmitir información menos familiar que la de la argumentación, se han localizado más errores léxicos.

La influencia positiva del conocimiento previo en la exactitud léxica de la producción escrita en LE, observada en el presente trabajo, se menciona también en el trabajo de Agustín Llach, Moreno Espinosa y Fernández Fontecha (2005). En este se detectó mayor densidad de errores léxicos en la narración, en la que los informantes tenían que contar una historia basada en una serie de viñetas, que en la descripción, en la que tenían que describir a su familia, es decir, debían transmitir información familiar y utilizar vocabulario conocido y frecuente. Sus resultados indican que la familiaridad con el tema favoreció la exactitud léxica de la producción escrita, tal como se afirma en el presente estudio.

Tedick (1990), por otra parte, mostró que el conocimiento previo puede ejercer influencia positiva en otros aspectos de la producción escrita. En concreto, los resultados arrojados por el estudio de Tedick (1990) indicaron una influencia positiva del conocimiento previo en la calidad y en la exactitud lingüística de la producción escrita de sus informantes que tenían un nivel intermedio y avanzado. De esta manera, y partiendo del supuesto de que la tarea argumentativa utilizada en el presente estudio era más fácil en el sentido de que los participantes tenían que emplear un vocabulario más familiar y frecuente, se puede asumir que el conocimiento previo tuvo un impacto en los resultados obtenidos en términos de grado de exactitud léxica alcanzado.

Respecto a la tercera pregunta de investigación, los resultados mostraron correlaciones significativas entre la fluidez y el número de errores solo en el caso de la tarea narrativa. Esta tendencia a la interacción se puede interpretar desde diferentes perspectivas. En primer lugar, en la condición allí y entonces los participantes se vieron obligados a recuperar y mantener en la memoria hechos (de la película que habían visto) antes de "contarlos en papel". Tal vez esta condición favoreciera únicamente a algunos aprendices, por ejemplo a aquellos con una mayor capacidad de memoria operativa de acuerdo a los postulados de Robinson. La necesidad de recuperar sucesos, guardarlos en la memoria operativa durante períodos temporales cortos e intentar verbalizarlos simultáneamente quizá haya provocado efectos de interferencia que, a su vez, condujeran a un procesamiento menos eficiente. Ello podría explicar en parte la interacción entre fluidez y exactitud léxica encontrada en la tarea narrativa.

Desde la perspectiva de los modelos de recursos inespecíficos por los que abogan Skehan (1996) y Skehan y Foster (2001), este resultado también sería esperable. Partiendo del supuesto de que la tarea narrativa es más compleja en el sentido de que el aprendiz tiene que utilizar recursos lingüísticos que todavía no han sido automatizados, o incluso cierto tipo de vocabulario menos familiar, es de esperar que se produzca una alteración de la atención. Esto conduciría a efectos de competición (trade-off), lo cual parece cierto dados los resultados del presente estudio.

Ahora bien, por lo que respecta a la tarea argumentativa, los resultados proporcionan una imagen diferente ya que no se encontraron correlaciones estadísticamente significativas 
entre la fluidez y la exactitud léxica. Para interpretar esta falta de asociación se puede atender a ciertos criterios. En primer lugar, las demandas léxicas de la tarea argumentativa utilizada se pueden considerar menos exigentes en comparación con las de la narración, ya que los informantes tuvieron que emplear un vocabulario más frecuente y familiar, relacionado con su vida diaria. Cabe añadir que el empleo del presente de indicativo probablemente funcionó como un factor facilitador, puesto que en un nivel B2 se ha practicado suficientemente este tiempo verbal, por lo que las exigencias de procesamiento de la lengua por parte de los informantes son menores. Todo lo anterior lleva a pensar que la tarea argumentativa tal vez no fuera tan difícil como para que se generaran efectos de competición entre los aspectos de la producción escrita examinados.

\section{Conclusiones}

Los resultados obtenidos en el presente estudio mostraron que el tipo de tarea escrita influye tanto en la fluidez como en la exactitud léxica. Asimismo, se comprobó que el conocimiento previo y la familiarización con el tema tiene una influencia positiva en la exactitud léxica de la producción escrita. Las implicaciones didácticas que derivan de los resultados del presente estudio pueden resultar útiles tanto para los docentes de ELE como para los diseñadores de material didáctico.

En primer lugar, queda claro que la familiarización con el tema de la tarea escrita puede estimular tanto la fluidez de la producción escrita como el léxico disponible de los estudiantes, de modo que cuenten con los recursos que les permitan expresar mejor sus ideas. Adicionalmente, la facilitación previa de vocabulario y de textos auténticos sobre el tema tratado puede tener una influencia positiva tanto en la fluidez de la producción escrita como en la exactitud léxica, dado que de esta manera se proporcionan ideas y vocabulario necesario para la producción escrita.

En cuanto a los profesionales que diseñan material didáctico y pruebas de evaluación de LE, se revela que la selección del tipo de tarea de producción escrita y del tópico es de importancia decisiva, ya que estos pueden condicionar la calidad del output de los estudiantes. En consecuencia, el examen cuidadoso de todas las características del tipo de tarea es indispensable, de forma que al diseñar tareas de producción escrita éstas mantengan el mismo nivel de dificultad y sean adecuadas para los diferentes grupos de aprendientes de ELE.

Pese a los resultados obtenidos es pertinente señalar una serie de limitaciones a las que se ha enfrentado el presente estudio. En primer lugar, cabe recordar que los índices empleados para medir los aspectos de la producción escrita examinados se consideran generales. Ello significa que el empleo de otros índices podría haber arrojado resultados diferentes. Otra limitación concierne al número limitado de participantes, que no permite la extrapolación de los resultados a toda la población de adultos aprendientes griegos de ELE. Se ha de advertir, además, que la imposibilidad de controlar otros factores, como la competencia estratégica o la competencia escrita en griego, la lengua materna de los informantes, no permite sacar conclusiones definitivas. Por último, debe señalarse que la selección de los temas de composición plantea una limitación metodológica adicional, dado que diferentes temas de narración o de argumentación podrían haber conducido a resultados distintos en términos de fluidez y exactitud léxica. 
Futuras investigaciones sobre la posible influencia del tipo de tarea en la producción escrita de la población meta (griegos aprendientes de ELE) serían imprescindibles. Se recomienda, pues, la repetición del presente estudio, incluyendo un mayor número de tipos de tarea de escritura y una evaluación más exhaustiva de su actuación, esto es, tras la inclusión de medidas de complejidad sintáctica y exactitud gramatical. Asimismo, futuros estudios que se centren en factores que influyen en la actuación de los aprendices de ELE, como la motivación, la percepción de la dificultad de la tarea o la capacidad de memoria operativa, permitirían un mejor entendimiento de la compleja interacción entre estos factores y el tipo de la tarea.

\section{REFERENCIAS BIBLIOGRÁFICAS}

Agustín Llach, M.P., Moreno Espinosa, S. y Fernández Fontecha, A. (2005). "Responding to different composition topics: a quantitative analysis of lexical error production", en Glosas Didacticas, 13: 128-140.

Carrell, P. y Connor, U. (1991). "Reading and writing descriptive and persuasive texts", in The Modern Language Journal, 75, 3: 314-324.

Consejo de Europa (2001). Marco común europeo de referencia para las lenguas: aprendizaje, enseñanza,evaluación. Madrid: MECD - Anaya.

Housen, A. y Kuiken, F. (2009). "Complexity, accuracy and fluency in second language acquisition", in Applied Linguistics, 30, 4: 461-473.

Larsen-Freeman, D. y Long, M. (1991). An introduction to second language research. London: Longman.

Rashid, S. y Rafik-Galea, S. (2007). "ESL writing variability: writing tasks, gender and proficiency level", in Indonesian Journal of English Language Teaching, 3, 2: 227-243.

Rezazadeh, M., Tavakoli, M. y Rasekh, A.E. (2011). "The role of task type in foreign language written production: focusing on fluency, complexity, and accuracy", in International Education Studies, 4, 2: 169-176.

Robinson, P. (2001). "Task complexity, task difficulty and task production: exploring interactions in a componential framework", in Applied Linguistics, 22, 1: 27-57.

Robinson, P. (2007). "Criteria for classifying and sequencing pedagogic tasks", in M. García Pelayo (ed.), Investigating tasks in Formal Language Learning. Clevedon: Multilingual Matters LTD, 7-26.

Serrano, R. S. (2011). "The effect of program type and proficiency level on learners' written production", in RESLA, 24: 211-226.

Simensen, A. (2010). "Fluency: an aim in teaching and a criterion in assessment", en Acta didactica Norge, 4, 1: 1-13.

Skehan, P. (1996). "A framework for the implementation of task - based instruction", in Applied Linguistics, 17, 1: 38-62.

Skehan, P. (1998). A cognitive approach to language learning. Oxford: Oxford University Press.

Skehan, P. y Foster, P. (1999). "The influence of task structure and processing conditions on narrative retellings", in Language Learning, 49, 1: 93-120.

Skehan, P. y Foster, P. (2001). "Cognition and tasks", en P. Robinson (ed.), Cognition and Second Language Instruction. Cambridge: Cambridge University Press, 183-205. 
Tarone, E. (1983). "On the variability of interlanguage systems", in Applied Linguistics, 4, 2 : 142-164.

Tedick, D. (1990). "ESL writing assessment: subject-matter knowledge and its impact on performance", in English for Specific Purposes, 9, 2: 123-143.

VanPatten, B. (1985). "The acquisition of ser and estar by adult learners of Spanish: a preliminary investigation of transitional stages of competence", in Hispania, 68, 2: 399-406.

VanPatten, B. (1989). "Can learners attend to form and content while processing input?", in Hispania, 72, 2: 409-417.

VanPatten, B. (1990). "Attending to form and content in the input: an experiment in consiousness", in Studies in Second Language Acquisition, 12: 287-301.

Way, D., Joiner, E y Seaman, M. (2000). "Writing in the secondary foreign language classroom: the effects of prompts and tasks on novice learners of French", in The Modern Language Journal, 84, 2: 171-184.

Wigglesworth, G. y Storch, N. (2009). "Pair versus individual writing: effects on fluency, complexity and accuracy", in Language Testing, 26, 3: 445-466.

Zhang, S. (1987). "Cognitive complexity and written production in English as a second language", in Language Learning, 37, 4: 469-481.

Centro Visual de Cervantes. Diccionario de términos clave de español como lengua extranjera. http://cvc.cervantes.es/ensenanza/biblioteca_ele/diccio_ele/diccionario/correccion.htm, fecha de consulta 4 de abril de 2013. 